\title{
The role of the iliotibial band cross-sectional area as a morphological parameter of the iliotibial band friction syndrome: a retrospective pilot study
}

\author{
Jiyeon Park ${ }^{1, *}$, Hyung Rae Cho ${ }^{2, *}$, Keum Nae Kang ${ }^{3}$, Kun Woong $\mathrm{Choi}^{3}$, Young Soon $\mathrm{Choi}^{1}$, Hye-Won Jeong ${ }^{1}$, \\ Jungmin $\mathrm{Yi}^{1}$, and Young Uk Kim${ }^{1}$ \\ 'Department of Anesthesiology and Pain Medicine, International St. Mary's Hospital, Catholic Kwandong University College of Medicine, Incheon, \\ Korea \\ ${ }^{2}$ Department of Anesthesiology and Pain Medicine, Myongji Hospital, Hanyang University College of Medicine, Goyang, Korea \\ ${ }^{3}$ Department of Anesthesiology and Pain Medicine, National Police Hospital, Seoul, Korea
}

Received October 19, 2020

Revised December 16, 2020

Accepted January 7, 2021

Handling Editor: Francis S. Nahm

\section{Correspondence}

Young Uk Kim

Department of Anesthesiology and

Pain Medicine, International St. Mary's

Hospital, Catholic Kwandong University

College of Medicine, 25 Simgok-ro

100beon-gil, Seo-gu, Incheon 22711,

Korea

Tel: +82-32-290-3570

Fax: +82-32-290-3568

E-mail: uk201@hanmail.net

*These authors contributed equally to this work.
Background: Iliotibial band friction syndrome (ITBFS) is a common disorder of the lateral knee. Previous research has reported that the iliotibial band (ITB) thickness (ITBT) is correlated with ITBFS, and ITBT has been considered to be a key morphologic parameter of ITBFS. However, the thickness is different from inflammatory hypertrophy. Thus, we made the ITB cross-sectional area (ITBCSA) a new morphological parameter to assess ITBFS.

Methods: Forty-three patients with ITBFS group and from 43 normal group who underwent T1W magnetic resonance imaging were enrolled. The ITBCSA was measured as the cross-sectional area of the ITB that was most hypertrophied in the magnetic resonance axial images. The ITBT was measured as the thickest site of ITB.

Results: The mean ITBCSA was $25.24 \pm 6.59 \mathrm{~mm}^{2}$ in the normal group and 38.75 $\pm 9.11 \mathrm{~mm}^{2}$ in the ITBFS group. The mean ITBT was $1.94 \pm 0.41 \mathrm{~mm}$ in the normal group and $2.62 \pm 0.46 \mathrm{~mm}$ in the ITBFS group. Patients in ITBFS group had significantly higher ITBCSA $(P<0.001)$ and ITBT $(P<0.001)$ than the normal group. A receiver operator characteristic curve analysis demonstrated that the best cut-off value of the ITBT was $2.29 \mathrm{~mm}$, with $76.7 \%$ sensitivity, $79.1 \%$ specificity, and area under the curve (AUC) 0.88. The optimal cut-off score of the ITBCSA was 30.66 $\mathrm{mm}^{2}$, with $79.1 \%$ sensitivity, $79.1 \%$ specificity, and AUC 0.87 .

Conclusions: ITBCSA is a new and sensitive morphological parameter for diagnosing ITBFS, and may even be more accurate than ITBT.

Key Words: Anatomy, Cross-Sectional; Cumulative Trauma Disorders; Diagnosis; Fascia Lata; Friction; Hypertrophy; Iliotibial Band Syndrome; Knee Injuries; Magnetic Resonance Imaging; ROC Curve; Sensitivity and Specificity.

\section{INTRODUCTION}

The iliotibial band (ITB) is a dense fibrotic anatomic struc- ture, originating in the iliac tubercle, and extending to the lateral aspect of the thigh, where the gluteus maximus, gluteus medius muscles, and fascia latae tensor insert, and (a) This is an open-access article distributed under the terms of the Creative Commons Attribution Non-Commercial License (http://creativecommons.org/licenses/by-nc/4.0/), which permits unrestricted non-commercial use, distribution, and reproduction in any medium, provided the original work is properly cited.

(C) The Korean Pain Society, 2021
Author contributions: Jiyeon Park: Writing/manuscript preparation; Hyung Rae Cho: Writing/manuscript preparation; Keum Nae Kang: Writing/manuscript preparation; Kun Woong Choi: Writing/manuscript preparation; Young Soon Choi: Data curation; Hye-Won Jeong: Methodology; Jungmin Yi: Computation; Young Uk Kim: Study conception. 
contributing to the monopodal equilibrium and postural control $[1,2]$. ITB lesions are frequently confused with pathologic conditions which cause discomfort on the lateral aspect of the knee, thigh, and hip. Iliotibial band friction syndrome (ITBFS) involves discomfort in the location of the lateral femoral condyle, that occurs after repetitive mechanical friction of the knee, typically in an athlete such as a runner or cyclist $[3,4]$.

A diagnosis is typically made based on the physical examination and case history, even though in a few cases magnetic resonance imaging (MRI) might be indicated to rule out another disease in the location $[5,6]$. An important positive sign on physical examination is local tenderness superior to the joint line and lateral knee inferior to the epicondyle. Flato et al. [1] have insisted that MRI findings in ITBFS mean increased signal intensity with fluid sequences both deep and superficial to the iliotibial (IT) tract origin, representing an edematous signal about the IT tract enthesis. MRI can also demonstrate partial tearing (intrinsic hyperintense signal) and thickening of the proximal IT tract attachments [1]. Ultrasonography (US) is an accurate, rapid, and widely available image modality capable of evaluating a wide spectrum of ITB alterations. Previous studies assessed the ITB thickness (ITBT) using a measurement at the approximate "halfway" point of the ITB [3]. However, an asymmetrical tear and partial thickening of the ITB can occur at any time [5]. Thus, measurement mistakes could occur routinely. In contrast to the ITBT, measuring a cross-sectional area of the ITB does not risk such measurement mistakes because the ITB crosssectional area (ITBCSA) measures a cross-sectional area of the whole ITB.

Therefore, to assess the hypertrophy of the whole ITB, we devised the ITBCSA as a new morphological imaging parameter. We assumed that the ITBCSA would be an excellent morphological imaging parameter for hypertrophied ITB. Thus, we used the knee MRI to compare the ITBCSA and ITBT between ITBFS patients and normal individuals.

\section{MATERIALS AND METHODS}

\section{Patients}

The Catholic Kwandong University institutional review board (IRB) approved this retrospective research protocol and waived the informed consent requirement (IRB approval number : IS20RISI0073). We performed a retrospective pilot study to investigate the role of the ITBCSA as a morphological parameter of the ITBFS. We reviewed ITBFS patients who visited International St. Mary's Hospital with knee pain from April 2014 to April 2020, and who had undergone knee MRI within 6 months of the first visit.

The inclusion criteria of ITBFS group were as follows [7,8]: 1) tenderness on palpation of the lateral knee approximately $2-3 \mathrm{~cm}$ above the join line; 2 ) typically localized pain just above the lateral part of the knee; 3 ) swelling of the outside of the knee; 4) knee pain during flexing the knee to thirty degrees; 5) gradual onset of symptoms and signs which, if they persisted for more than 1 month, could cause interference in daily activities or in sports participation; 6) knee pain that worsens with repetitive activities or continuing to participate in running. We excluded patients if they had any of the following problems: 1) history of knee surgery; 2) osteosarcoma; 3) history of the femur or the tibial surgery; and 4) meniscus or lateral collateral ligament injury. Forty-three patients were enrolled. There were 21 $(60.4 \%)$ males and 22 (39.6\%) females with an average age of $47.5 \pm 17.3$ years (range, 16 to $66 \mathrm{yr}$ ) (Table 1). To compare the ITBCSA and ITBT between patients and normal individuals, we also enrolled a healthy group. The normal group was people who had a knee MRI examination due to knee discomfort, however, no structural problems were found in the MRI. In the normal group, 43 individuals (21 males and 22 females) were enrolled with an average age of $47.6 \pm 14.3$ years (range, 15 to $68 \mathrm{yr}$ ).

Table 1. Comparison of the characteristics of control and ITBFS groups

\begin{tabular}{lccc}
\hline \multicolumn{1}{c}{ Variable } & Control group $(\mathrm{n}=43)$ & ITBFS group $(\mathrm{n}=43)$ & $P$ value \\
\hline Sex $($ male/female) & $21 / 22$ & $26 / 17$ & 0.279 \\
Age $(\mathrm{yr})$ & $47.6 \pm 14.3$ & $46.3 \pm 11.5$ & 0.317 \\
Height $(\mathrm{cm})$ & $164.5 \pm 12.3$ & $166.8 \pm 9.0$ & 0.328 \\
Weight $(\mathrm{kg})$ & $69.9 \pm 13.7$ & $74.8 \pm 13.4$ & 0.884 \\
Duration of illness $(\mathrm{mo})$ & - & $9.9 \pm 17.4$ & - \\
ITBT $(\mathrm{mm})$ & $1.9 \pm 0.4$ & $2.6 \pm 0.5$ & $<0.001$ \\
ITBCSA $\left(\mathrm{mm}^{2}\right)$ & $25.2 \pm 6.6$ & $38.8 \pm 9.1$ & $<0.001$ \\
\hline
\end{tabular}

Values are presented as number only or mean \pm standard deviation.

ITBFS: iliotibial band friction syndrome, ITBT: iliotibial band thickness, ITBCSA: iliotibial band cross-sectional area. 

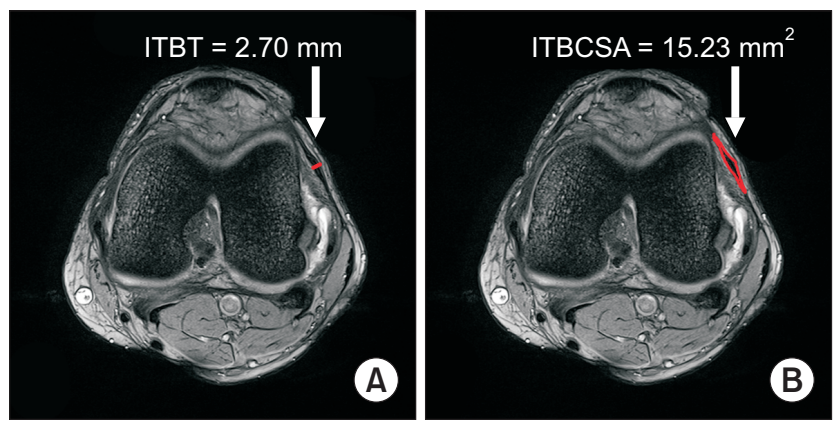

Fig. 1. Measurement of both iliotibial band thickness (ITBT) (white arrow) (A) and iliotibial band cross-sectional area (ITBCSA) (white arrow) (B) in the iliotibial band friction syndrome group was carried out on magnetic resonance $\mathrm{T} 1$ weighted images.

\section{Imaging parameters}

Magnetom Skyra MRI analysis was done using a 3.0-T MRI (Siemens Healthcare GmbH, Erlangen, Germany) and 3.0T Ingenia scanners (Philips, Eindhoven, The Netherlands). Knee MRI examinations performed in standard supine position under non-weight-bearing conditions. For all MRI examinations, we acquired coronal and axial T1W images with a slice thickness of $3.0 \mathrm{~mm}$, intersection gap of 0.9 $\mathrm{mm}$, repetition time/echo time of $492 \mathrm{~ms} / 15 \mathrm{~ms}, 160 \times 160$ $\mathrm{cm}$ field of view, $320 \times 316$ pixels of scanning matrix, and > 3 echo train length.

\section{Image analysis}

ITBCSA and ITBT measurements were performed by the same specialist. All images were reviewed blindly. We acquired axial TIW magnetic resonance images at the thickest visualization of the ITB. We measured the ITBCSA and ITBT on MRI using an image analysis system (INFINITT PACS; INFINITT Healthcare, Seoul, Korea) (Fig. 1). The ITBCSA was measured as the tendon cross-sectional area of the ITB at the thickest point. The cross-sectional area is automatically presented by the "Measure area free hand" tool in INFINITT PACS after drawing a line along the border of the ITB. The ITBT was measured at the most hypertrophied ITB.

\section{Statistical analysis}

We compared the ITBCSA and ITBT between the ITBFS and the control groups using independent $t$-tests. The validity of the ITBCSA and ITBT in the diagnosis of hypertrophy was measured by the receiver operator characteristic (ROC) curve with the area under the curve (AUC)-index. A probability value under 0.05 was considered statistically significant. All analyses were processed by using SPSS

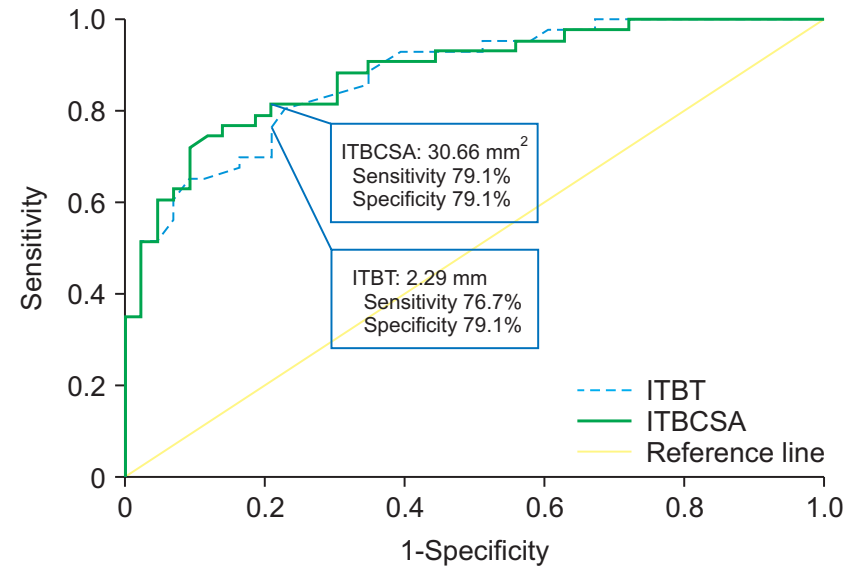

Fig. 2. Receiver operating characteristic (ROC) curve of iliotibial band thickness (ITBT) and iliotibial band cross-sectional area (ITBCSA) for prediction of iliotibial band friction syndrome (ITBFS). The best cut off point of ITBT was $2.29 \mathrm{~mm}$ versus $30.66 \mathrm{~mm}^{2}$ of ITBCSA, with sensitivity $76.7 \%$ vs. $79.1 \%$, specificity $79.1 \%$ vs. $79.1 \%$ and AUC 0.87 vs. 0.88 , respectively. ITBT area under the curve (AUC) (95\% confidence interval [CI]) $=0.87(0.80-0.94)$, ITBCSA AUC $(95 \% \mathrm{Cl})=0.88(0.81-0.95)$.

Table 2. Sensitivity and specificity of each cut-off point of the ITBT

\begin{tabular}{ccc}
\hline ITBT $(\mathrm{mm})$ & Sensitivity $(\%)$ & Specificity $(\%)$ \\
\hline 1.21 & 100.0 & 4.7 \\
1.70 & 97.7 & 32.6 \\
1.82 & 97.7 & 39.5 \\
2.03 & 93.0 & 60.5 \\
2.17 & 83.7 & 69.8 \\
$2.29^{\mathrm{a}}$ & 76.7 & 79.1 \\
2.41 & 69.8 & 83.7 \\
2.53 & 55.8 & 93.0 \\
2.77 & 34.9 & 97.7 \\
2.82 & 32.6 & 100.0 \\
\hline
\end{tabular}

ITBT: iliotibial band thickness.

${ }^{\text {a }}$ The optimal cut-off score on the receiver operating characteristic curve.

software, version 22.0 (IBM Co., Armonk, NY).

\section{RESULTS}

The mean ITBCSA was $25.2 \pm 6.6 \mathrm{~mm}^{2}$ in the normal group and $38.8 \pm 9.1 \mathrm{~mm}^{2}$ in the ITBFS group. The mean ITBT was $1.9 \pm 0.4 \mathrm{~mm}$ in the normal group and $2.6 \pm 0.5 \mathrm{~mm}$ in the ITBFS group. Patients in ITBFS group had significantly higher ITBCSA $(P<0.001)$ and ITBT $(P<0.001)$ than the normal group (Table 1). A ROC curve analysis demonstrated that the most suitable cut-off value of the ITBT was 2.29 $\mathrm{mm}$, with $76.7 \%$ sensitivity and $79.1 \%$ specificity. The AUC of the parametric ROC curve was 0.88 (95\% confidence interval [CI], 0.81-0.95) (Fig. 2, Table 2). The most suitable 
Table 3. Sensitivity and specificity of each cut-off point of the ITBCSA

\begin{tabular}{ccc}
\hline ITBCSA $\left(\mathrm{mm}^{2}\right)$ & Sensitivity $(\%)$ & Specificity $(\%)$ \\
\hline 13.17 & 100.0 & 2.3 \\
17.32 & 100.0 & 11.6 \\
21.51 & 97.7 & 27.9 \\
24.29 & 93.0 & 46.5 \\
27.76 & 88.4 & 65.1 \\
$30.66^{a}$ & 79.1 & 79.1 \\
33.14 & 72.1 & 90.7 \\
36.27 & 60.5 & 93.0 \\
38.62 & 51.2 & 97.7 \\
41.99 & 34.9 & 100.0 \\
\hline
\end{tabular}

ITBCSA: iliotibial band cross-sectional area.

${ }^{\text {a}}$ The optimal cut-off score on the receiver operating characteristic curve.

cut-off score for the ITBCSA was $30.66 \mathrm{~mm}^{2}$, with $79.1 \%$ sensitivity and $79.1 \%$ specificity, and the AUC index was 0.87 (95\% CI, 0.80-0.94) (Fig. 2, Table 3).

\section{DISCUSSION}

ITBFS is a very common overuse soft-tissue trauma of the knee that occurs as a result of mechanical repetitive injury [9-11]. The ITB is a thick fascia strip that originates from the iliac crest tubercle, continues to the lateral side of the thigh, and inserts into the lateral proximal fibular head and into the lateral tibial condyle [1]. ITBFS generates when friction occurs between the underlying lateral femoral epicondyle and the posterior edge of the ITB. When the knee is extended, the ITB moves anterior to this landmark; however, when the knee is flexed to an angle greater than thirty degrees, the ITB lies posterior to the lateral femoral epicondyle. Thus, friction occurs at slightly less than thirty degrees of knee flexion, when the ITB crosses over the lateral femoral epicondyle. The cumulative effect of mechanical friction causes an inflammatory disorder within the periosteum of the lateral femoral epicondyle, underlying bursa, and iliotibial tract, eventually inducing lateral knee pain [11-15]. However, several other diseases may also be observed in this area, both distally and proximally in relation to the usually affected location in the lateral knee [13]. Thus, exact diagnosis is very important.

Various diagnostic imaging technique, such as US, MRI, radiography, stress radiography, and computed tomography are available $[3,6]$, however, the diagnosis of ITBFS is still not easy due to the lack of an exact morphological image parameter. The process of ITB thickening starts with continuous mechanical stress, which induces inflammation, structural damage, and finally thickening $[2,16,17]$.

We assumed that the cross-sectional area of the ITB can predict hypertrophy of the ITB because the ITBCSA does not suffer from this measurement mistake since the ITBCSA measures the cross-sectional area of the whole ITB, in contrast to the ITBT [18]. We eventually concluded that the ITBCSA is better than the ITBT as a morphological image parameter of ITBFS. In this research, we found that the ITBCSA had $79.1 \%$ sensitivity and $79.1 \%$ specificity. The AUC-index for the ROC curve was 0.88 (95\% CI, 0.81-0.95) to predict ITBFS. In contrast, the ITBT had $76.7 \%$ sensitivity and $79.1 \%$ specificity. The AUC-index for the ROC curve was 0.87 (95\% CI, 0.80-0.94). These results show that the ITBCSA is a better morphological predictor of ITBFS than the ITBT. To our knowledge, this is the first research evaluating the accuracy of ITBCSA in the diagnosis of ITBFS.

There are some limitations to the current research. First, alternative image modalities to assess ITBFS, such as US and radiography, have been proved to be effective at diagnosing ITBFS [3]. Especially, US is a rapid, and widely available image modality [19-22]. However, the current study only evaluated the measurement of the ITBCSA and ITBT on MRI. Second, the small sample size may have limited our conclusion to detect statistically meaningful differences. Third, ITBFS has multiple causes, including the swelling of the bursa, bowed legs, and alteration in activity levels. However, we only focused on ITBT and the ITBCSA. Fourth, this research is retrospective in nature.

In conclusion, ITBCSA is a new and sensitive morphological parameter for diagnosing ITBFS, and may even be more accurate than ITBT. When evaluating the ITBFS in patients with recurrent knee pain, physicians should carefully assess the ITBCSA rather than the ITBT. ITBCSA may be a new objective indicator of ITBFS.

\section{CONFLICT OF INTEREST}

No potential conflict of interest relevant to this article was reported.

\section{FUNDING}

No funding to declare.

\section{ORCID}

Jiyeon Park, https://orcid.org/0000-0002-1727-2411

Hyung Rae Cho, https://orcid.org/0000-0003-1634-7482

Keum Nae Kang, https://orcid.org/0000-0002-1135-6403

Kun Woong Choi, https://orcid.org/0000-0002-3880-304X

Young Soon Choi, https://orcid.org/0000-0002-3819-0271 
Hye-Won Jeong, https://orcid.org/0000-0002-8286-5715

Jungmin Yi, https://orcid.org/0000-0003-4481-8923

Young Uk Kim, https://orcid.org/0000-0003-4977-5272

\section{REFERENCES}

1. Flato R, Passanante GJ, Skalski MR, Patel DB, White EA, Matcuk GR Jr. The iliotibial tract: imaging, anatomy, injuries, and other pathology. Skeletal Radiol 2017; 46: 605-22.

2. Hadeed A, Tapscott DC. Iliotibial band friction syndrome [Internet]. Treasure Island (FL): StatPearls Publishing; 2020. Available at: https://www.ncbi.nlm.nih.gov/books/ NBK542185/.

3. Hong JH, Kim JS. Diagnosis of iliotibial band friction syndrome and ultrasound guided steroid injection. Korean J Pain 2013; 26: 387-91.

4. Lavine R. Iliotibial band friction syndrome. Curr Rev Musculoskelet Med 2010; 3: 18-22.

5. Lee S, Cho HR, Yoo JS, Kim YU. The prognostic value of median nerve thickness in diagnosing carpal tunnel syndrome using magnetic resonance imaging: a pilot study. Korean J Pain 2020; 33: 54-9.

6. Yan R, Huang Z, Wang L, Zhang X. [MR manifestations and clinical significance of iliotibial band friction syndrome]. Zhonghua Yi Xue Za Zhi 2014; 94: 1473-5. Chinese.

7. Khaund R, Flynn SH. Iliotibial band syndrome: a common source of knee pain. Am Fam Physician 2005; 71: 1545-50.

8. Strauss EJ, Kim S, Calcei JG, Park D. Iliotibial band syndrome: evaluation and management. J Am Acad Orthop Surg 2011; 19: 728-36.

9. Rathore S, Quadri V, Tapadia S, Krishnaiah K, Krishna VPN. Intra-articular fibroma of tendon sheath in knee joint associated with iliotibial band friction syndrome: rare occurrence in a teenage girl. J Orthop Case Rep 2017; 7: 28-31.

10. Takagi K, Inui H, Taketomi S, Yamagami R, Kono K, Nakazato $\mathrm{K}$, et al. Iliotibial band friction syndrome after knee arthroplasty. Knee 2020; 27: 263-73.

11. Walbron P, Jacquot A, Geoffroy JM, Sirveaux F, Molé D. Iliotibial band friction syndrome: an original technique of digastric release of the iliotibial band from Gerdy's tubercle. Orthop Traumatol Surg Res 2018; 104: 1209-13.

12. Decker G, Hunt D. Proximal iliotibial band syndrome in a runner: a case report. PM R 2019; 11: 206-9.
13. Everhart JS, Kirven JC, Higgins J, Hair A, Chaudhari AAMW, Flanigan DC. The relationship between lateral epicondyle morphology and iliotibial band friction syndrome: a matched case-control study. Knee 2019; 26: 1198-203.

14. Foch E, Milner CE. Influence of previous iliotibial band syndrome on coordination patterns and coordination variability in female runners. J Appl Biomech 2019; 35: 305-11.

15. Landreau P, Catteeuw A, Hamie F, Saithna A, SonneryCottet B, Smigielski R. Anatomic study and reanalysis of the nomenclature of the anterolateral complex of the knee focusing on the distal iliotibial band: identification and description of the condylar strap. Orthop J Sports Med 2019; 7: 2325967118818064.

16. Pegrum J, Self A, Hall N. Iliotibial band syndrome. BMJ 2019; 364: 1980.

17. Everhart JS, Kirven JC, Higgins J, Hair A, Chaudhari AMW, Flanigan DC. Corrigendum to "The relationship between lateral epicondyle morphology and iliotibial band friction syndrome: A matched case-control study [The Knee 26 (2019) 1198-1203]". Knee 2020; 27: 1291.

18. Joo Y, Cho HR, Kim YU. Evaluation of the cross-sectional area of acromion process for shoulder impingement syndrome. Korean J Pain 2020; 33: 60-5.

19. Gadsden JC, Sata S, Bullock WM, Kumar AH, Grant SA, Dooley JR. The relative analgesic value of a femoral nerve block versus adductor canal block following total knee arthroplasty: a randomized, controlled, double-blinded study. Korean J Anesthesiol 2020; 73: 417-24.

20. Ciftci B, Ekinci M, Atalay YO. Ultrasound guided rhomboid intercostal block provides effective pain control after videoassisted thoracoscopic surgery: a brief report of three cases. Korean J Anesthesiol 2020. doi: 10.4097/kja.20538.

21. Abdelbaser I, Mageed NA, El-Emam EM, ALseoudy MM, Elmorsy MM. Preemptive analgesic efficacy of ultrasoundguided transversalis fascia plane block in children undergoing inguinal herniorrhaphy: a randomized, double-blind, controlled study. Korean J Anesthesiol 2020. doi: 10.4097/ kja.20601.

22. Mudumbai SC, Kim TE, Howard SK, Giori NJ, Woolson S, Ganaway T, et al. Corrigendum: an ultrasound-guided fascia iliaca catheter technique does not impair ambulatory ability within a clinical pathway for total hip arthroplasty. Korean J Anesthesiol 2020; 73: 267. 